\title{
PHOTOIONIZATION OF EXCIMERS PRODUCED IN NAPHTHALENE CLUSTERS
}

\author{
HIROYUKI SAIGUSA \\ Department of Chemistry, The University of Akron, Akron, Ohio 44325-3601
}

(Received 14 March 1993)

\begin{abstract}
The excited-state dynamics of small naphthalene clusters that occur upon selective excitation into $S_{1}$ vibronic levels have been investigated by mass-selective pump-probe photoionization spectroscopy. The results provide direct evidence for structural isomerization of the initially excited van der Waals cluster into an excimer geometry, consistent with the fluorescence results. The excimer has been identified as a cluster having a strongly bound dimer core surrounded by ground-state molecules, thus ruling out the possibility of excimer formation via monomer evaporation. We have also found that ionization is enhanced when the initially excited cluster undergoes excimer formation, which provides a new ionization mechanism for aromatic clusters. The ionization enhancement is explained by the Franck-Condon factors associated with the ionizing transition from the excimer state.
\end{abstract}

KEY WORDS: Naphthalene cluster, MPI, two color photoionization, excimer formation, cooperative ionization by excimer formation, vdW cluster

\section{INTRODUCTION}

The evolution of dynamical properties in clusters as a function of size is a fundamental question, the investigation of which is expected to provide direct insight into the transition of characteristics from molecular scale to bulk systems. Whereas such size dependence can now be obtained for clusters involving atoms or small molecules, ${ }^{1}$ the situation is much worse for clusters of large molecules. One reason for this is the lack of effective methods for producing higher clusters of less volatile molecules such as polycyclic aromatic compounds. Thus, benzene clusters have frequently been selected as objects of spectroscopic investigations.

Naphthalene is also highly favorable for such studies. First, the resonance intermolecular (exciton) interactions between naphthalene molecules in the $S_{1}$ state were measured in the bulk crystalline system using elegant low-temperature spectroscopy. ${ }^{2}$ Second, well-resolved $S_{1}$ spectra were also recorded for cold van der Waals (vdW) clusters. ${ }^{3,4}$ The multiple discrete components observed for the vibronic transitions suggested the occurrence of excitonic effects in these clusters. Moreover, these clusters display strong evidence for dynamical process (excimer formation) when excited into $S_{1}$ vibronic levels. ${ }^{5,6}$ The unique relationship between the cluster size 
and excimer formation efficiency suggested that the dynamics is geometrically controlled. The higher clusters, particularly the trimer, require substantial activation energies toward the excimer formation while the dimer is nearly free to rearrange to an excimer geometry. Although the possibility of such a dynamical process was suggested for the benzene dimer, ${ }^{7}$ no direct evidence was obtained. ${ }^{8}$

In a previous publication, ${ }^{9}$ we demonstrated, using a photodissociation technique, that the excimer generated upon excitation of the naphthalene clusters $\left[\left(\mathrm{C}_{10} \mathrm{H}_{8}\right)_{\mathrm{n}}\right.$ $(\mathrm{n}=3,4)$ ], exhibits a strong absorption band in the red and near IR regions. Excitation into this band was found to cause rapid photodissociation of the excimer, thus producing monomer fragments. Based on this photodissociation behavior, the stability of the excimer was interpreted by the exciton-resonance interactions between the monomer $\mathrm{S}_{2}$ states, with significant contributions from the charge-resonance interactions. However, it was not possible in this study to determine the number of naphthalene molecules involved in these excimers, and simply assumed that the excimer is a tightly bound dimeric species obtained upon ejection of ground-state monomers: $\left(\mathrm{C}_{10} \mathrm{H}_{8}\right)_{\mathrm{n}}{ }^{*} \rightarrow\left(\mathrm{C}_{10} \mathrm{H}_{8}\right)_{2}{ }^{*}+(\mathrm{n}-2)\left(\mathrm{C}_{10} \mathrm{H}_{8}\right)$. In the work reported here, we present the results of a careful search for cluster ions produced upon excitation of the naphthalene clusters $\left[\left(\mathrm{C}_{10} \mathrm{H}_{8}\right)_{n}(\mathrm{n}=2-4)\right]$ in a two-color resonant two-photon ionization (R2PI) scheme, with a special emphasis on the identification of the excimers produced in these clusters.

Another object in the present work is to understand a quantitative relationship between excimer formation in aromatic clusters and their photoionization dynamics. Schriver et al. ${ }^{10}$ discovered that large benzene clusters ionize with anomalously high efficiency compared to the monomer. The ionization enhancement was explained by an exciton annihilation mechanism involving sequential excitation of two or more molecules in the cluster. Subsequently, direct spectroscopic evidence for the existence of such a cooperative ionization process was reported for the naphthalene trimer, ${ }^{11}$ which demonstrated that the ionization signal increases whenever both pump and ionizing laser beams are tuned to the vicinity of a specific $S_{1}$ vibronic transition. Both results appear to favor the exciton annihilation mechanism for the photoionization of aromatic clusters.

This study also investigates the photoionization dynamics of the naphthalene cluster near the threshold region using the two-color pump-probe photoionization technique. For all the clusters studied $(n=2-4)$, the two-color ionization occurs well below the monomer ionization potential (IP) of $65,664 \mathrm{~cm}^{-1}(8.14 \mathrm{eV}),{ }^{12}$ indicating that these clusters ionize via typical $\left(1+1^{\prime}\right)$ ionization. We show here compelling evidence that cluster ionization efficiency increases as the initially prepared vdW cluster rearranges to the excimer. The ionization enhancement is accounted for by invoking structural similarity between the excimer and ionic cluster.

\section{EXPERIMENTAL}

The experimental arrangement and procedures were essentially the same as those described previously. ${ }^{5}$ Briefly, the apparatus consists of a pulsed molecular beam 
and a linear time-of-flight (TOF) mass spectrometer. The naphthalene clusters were produced by supersonic expansion at 50 -psi He through a nozzle having a diameter of $0.5 \mathrm{~mm}$. The jet was skimmed by a 2-mm nickel skimmer, and the resulting cluster beam passed into an ionization chamber maintained at $<2 \times 10^{-6}$ Torr, where, after a 10-cm flight, it intersected the frequency-doubled output of a YAG-pumped dye laser (Quanta Ray DCR-1/Lambda Physik FL-2002). The first laser was used to excite a parent vdW cluster into a resonant $S_{1}$ state. The doubled output of a second YAG-pumped dye laser (Continuum NY61-10/Quanta Ray PDL-1 + WEX-1), timedelayed by $<500 \mathrm{~ns}$, was employed to cause ionization either from the initially prepared state or from the resulting excimer state. The ions produced were accelerated in an electric field of $315 \mathrm{~V} / \mathrm{cm}$, and drifted $150 \mathrm{~cm}$ to a dual microchannel plate detector. Under the ion extraction condition, a field shift of $\approx 50 \mathrm{~cm}^{-1}$ was observed for the monomer ionization. The time-resolved ion signals were digitized and accumulated by a $400-\mathrm{MHz}$ transient digitizer (Tektronix 11402). We probed the excited-state dynamics of each cluster by monitoring the two-color photoion signal as a function of the time delay between the pump and probe (ionizing) laser pulses. The resulting time-resolved data were influenced only by pulse-to-pulse timing jitter $( \pm 2 \mathrm{~ns})$ of each pulse (3-ns width). In order to minimize one-color signals, the pump laser was kept at a low fluence of $<0.1 \mathrm{~mJ} /$ pulse while higher fluences of $0.1-0.4$ $\mathrm{mJ} /$ pulse were used for the ionizing transition. Photoionization efficiency spectra were recorded, at a fixed pump laser wavelength, by scanning the ionizing laser in the wavelength region of $337.5-315 \mathrm{~nm}$.

\section{RESULTS}

\section{A. Ionization through $\overline{8}_{0}^{I}$ intermediate}

As reported earlier, ${ }^{5}$ the tetramer $\left(\mathrm{C}_{10} \mathrm{H}_{8}\right)_{4}$ excited into the most intense $\mathrm{S}_{1}$ band (denoted $\overline{8}_{0}^{1}$ using the notation of Stockburger et al. ${ }^{13}$ ) undergoes excimer formation, giving rise to a strongly red-shifted fluorescence centered near $380 \mathrm{~nm}$. Based on the time-resolved fluorescence measurements, the rearrangement time has been estimated to be $<3 \mathrm{~ns}^{6}{ }^{6}$ This predicts that ionizing transitions occur from the excimer state in the nanosecond pump-probe scheme employed in this study. Figure 1 shows the two-color TOF spectra of $\left(\mathrm{C}_{10} \mathrm{H}_{8}\right)_{4}^{+}$, recorded at different pump-probe delays in the range 3 to $465 \mathrm{~ns}$. These spectra are obtained when the pump laser is tuned to the most intense spectral feature (at $\left.32,304 \mathrm{~cm}^{-1}\right)$ for $\overline{8}_{0}^{1}$ of $\left(\mathrm{C}_{10} \mathrm{H}_{8}\right)_{4}$, followed by the ionizing laser at $321 \mathrm{~nm}$. This indicates that the two-color enhancement occurs at energies well below the one-color two-photon $(1+1)$ ionization limit.

The temporal behavior of the two-color signal is plotted as a function of the time delay in Figure 2 . The two-color signal decays roughly exponentially with a 300-ns lifetime. Also shown is the decay curve of the excimer fluorescence resulting from the pump laser excitation. Its lifetime has been determined to be $340 \mathrm{~ns}^{6}{ }^{6}$ Direct correspondence of the temporal behaviors of the $\left(\mathrm{C}_{10} \mathrm{H}_{8}\right)_{4}^{+}$signal and the excimer 


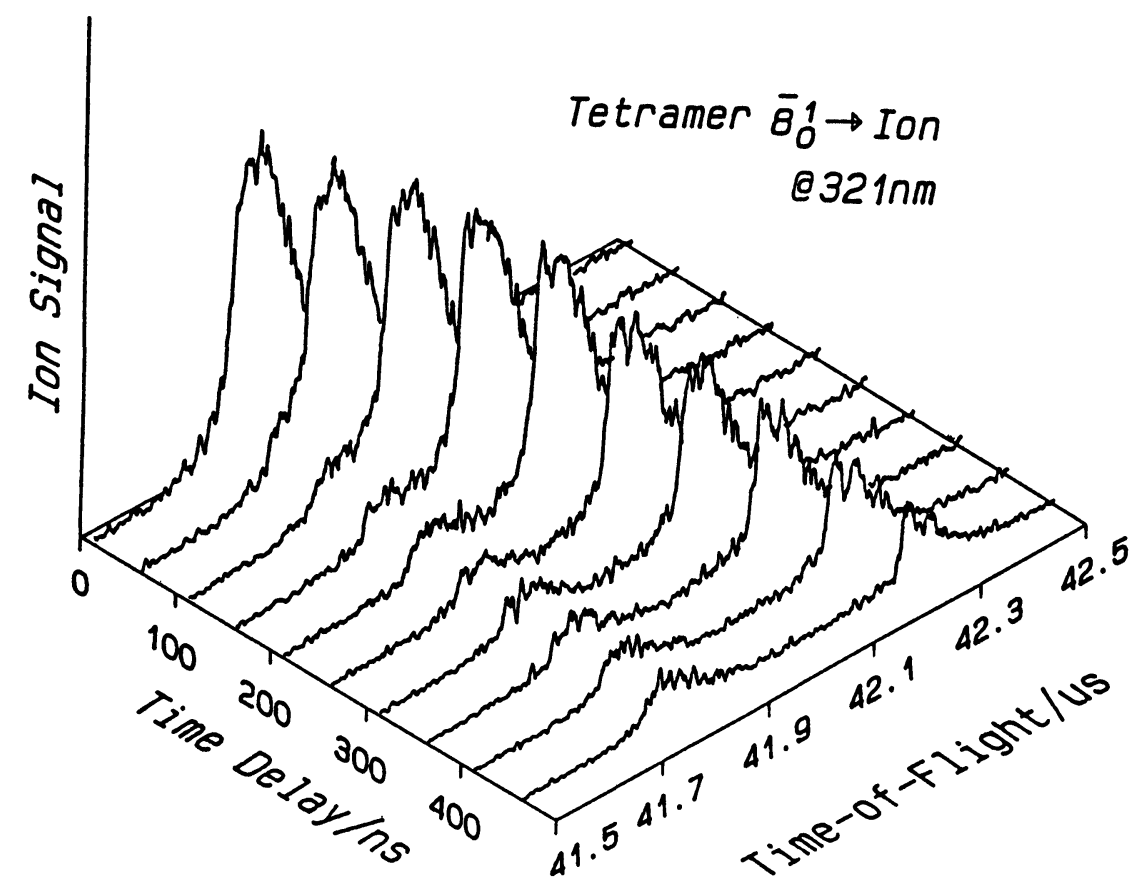

Figure 1 Two-color TOF mass spectra of the naphthalene tetramer ion $\left(\mathrm{C}_{10} \mathrm{H}_{8}\right)_{4}^{+}$obtained as a function of the time delay between the pump and ionizing laser pulses. The tetramer cluster is excited at the strongest peak $\left(32,304 \mathrm{~cm}^{-1}\right)$ in the $\overline{8}_{0}^{1}$ region, and then ionized with a $321-\mathrm{nm}$ photon.

fluorescence is evident. This demonstrates that the excimer consists of the same number of naphthalene molecules as in the parent cluster, and the $\left(\mathrm{C}_{10} \mathrm{H}_{8}\right)_{4}^{+}$ion observed here arises from the ionization of the excimer. Attempts were also made to search for $\left(\mathrm{C}_{10} \mathrm{H}_{8}\right)_{2}^{+}$ion under the same pump-probe configuration. Although $\left(\mathrm{C}_{10} \mathrm{H}_{8}\right)_{2}^{+}$ions were observed in the TOF spectra, we found that these ions are due to direct excitation of the neutral dimer. It should be noted that the dimer exhibits a broad absorption feature which encompasses the $\overline{8}_{0}^{1}$ band of $\left(\mathrm{C}_{10} \mathrm{H}_{8}\right)_{4}{ }^{5}$ thus both clusters are excited at the pump laser wavelength employed here. These findings rule out the possibility of excimer formation involving evaporation of ground-state monomers.

Previously, the one-color $(1+1)$ ionization spectra of the naphthalene clusters in the $\overline{8}_{0}^{1}$ region were reported by Wessel and Syage, ${ }^{3,4}$ and by our group. ${ }^{5}$ Figure 3 displays the corresponding $\overline{8}_{0}^{1}$ spectra recorded by the two-color $\left(1+1^{\prime}\right)$ ionization method. These spectra were obtained, at a fixed ionizing wavelength of $320 \mathrm{~nm}$, while scanning the pump laser across the $\overline{8}_{0}^{1}$ region. In each case, the spectral features appearing in the two-color spectrum match well those of the one-color spectrum. This indicates that the cluster ions observed here originate from the corresponding neutral clusters, thus excluding the possibility of metastable dissociation upon photoionization. However, it should be noted that the dimer spectrum in Figure 3(a) exhibits a sharp peak located at $-81 \mathrm{~cm}^{-1}$, which coincides well with the strongest 


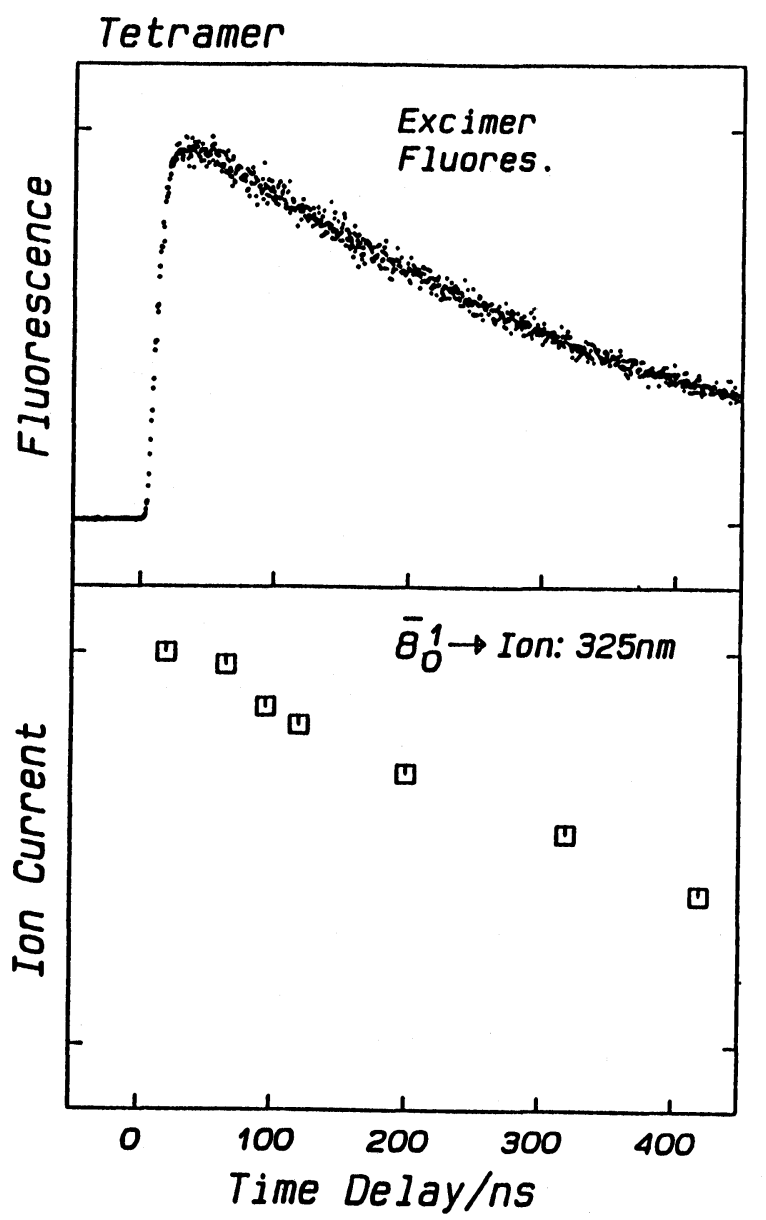

Figure 2 Comparison of temporal behaviors for the two-color signal of $\left(\mathrm{C}_{10} \mathrm{H}_{8}\right)_{4}^{+}$(lower panel) and for the excimer fluorescence (upper panel). The two-color signal is plotted as a function of the time delay between the pump laser pulse, fixed at $\overline{8}_{0}^{1}$ of the tetramer, and the ionizing laser pulse at $325 \mathrm{~nm}$. The excimer fluorescence resulting from the same pump laser excitation is detected at $>400 \mathrm{~nm}$.

peak of $\left(\mathrm{C}_{10} \mathrm{H}_{8}\right)_{3}$. The $\left(\mathrm{C}_{10} \mathrm{H}_{8}\right)_{2}^{+}$ion signal at $-81 \mathrm{~cm}^{-1}$ is caused by the pump laser alone, indicating that the $\left(\mathrm{C}_{10} \mathrm{H}_{8}\right)_{3}^{+}$ion produced via $(1+1)$ ionization favors a metastable dissociation into $\left(\mathrm{C}_{10} \mathrm{H}_{8}\right)_{2}^{+}$.

The photoionization efficiency curves observed for $\left(\mathrm{C}_{10} \mathrm{H}_{8}\right)_{8}^{+}(\mathrm{n}=2-4)$, in the total energy region of $62,000-63,500 \mathrm{~cm}^{-1}$, taken at a pump-probe delay of $200 \mathrm{ns, \text {are }}$ compared in Figure 4. The pump laser was fixed at the most intense feature of the $\overline{8}_{0}^{1}$ resonance of each cluster appearing in Figure 3. In all cases, the two-color signal intensity increases gradually as the ionization laser is scanned to higher energies. However, there are some important differences among these spectra. The most striking is that the $\left(\mathrm{C}_{10} \mathrm{H}_{8}\right)_{4}^{+}$spectrum displays a long tail extending below $62,000 \mathrm{~cm}^{-1}$. 


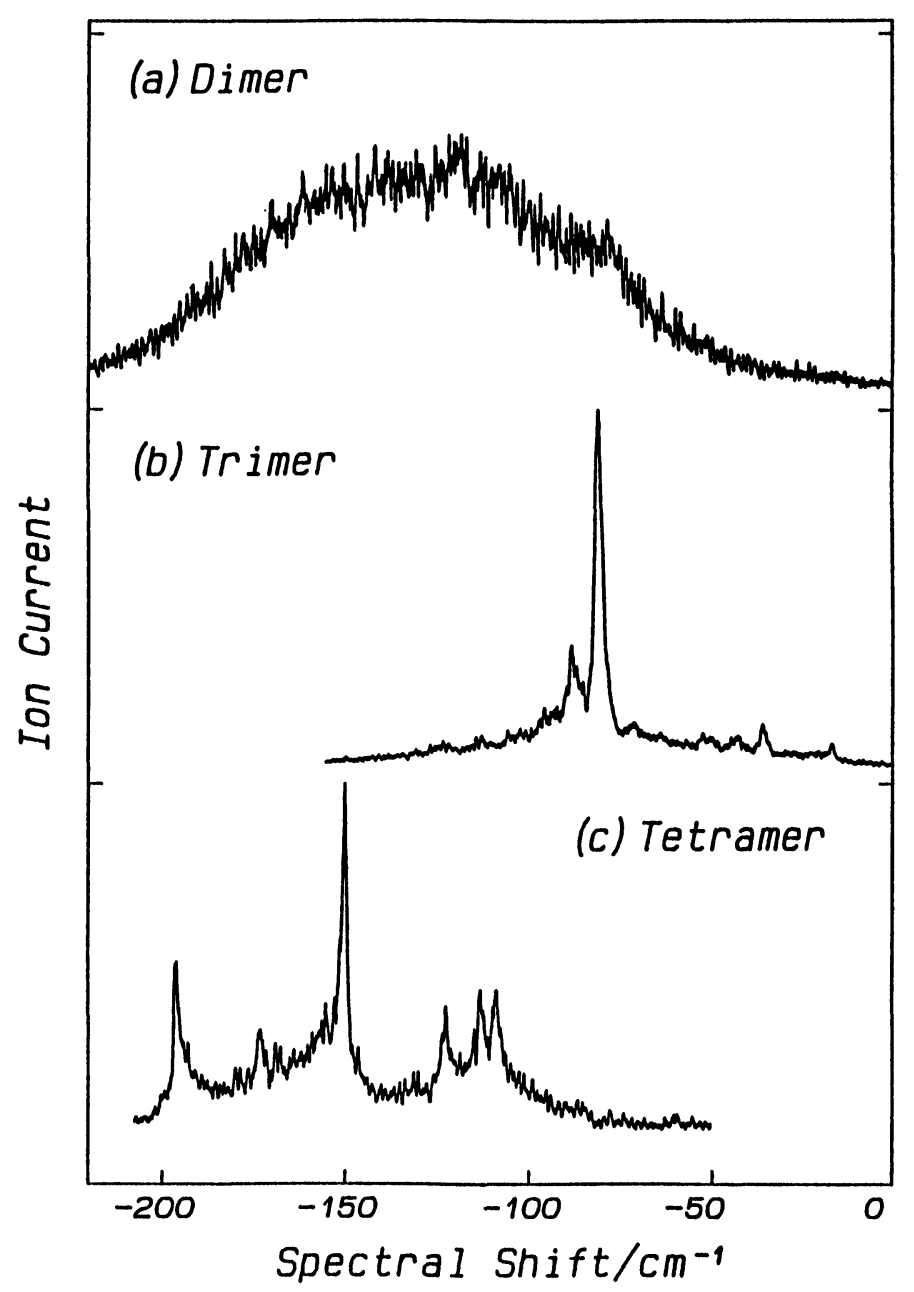

Figure 3 Two-color R2PI spectra of (a) the dimer, (b) the trimer, and (c) the tetramer in the $\overline{8}_{0}^{1}$ region. The ionizing laser, fixed at $320 \mathrm{~nm}$, fires at a $200 \mathrm{~ns}$ time delay with respect to the pump laser. The spectral shift is relative to the monomer $\overline{8}_{0}^{1}$ transition at $32,455 \mathrm{~cm}^{-1}$.

The lowest value measured in this experiment is $61,900 \mathrm{~cm}^{-1}$; this corresponds to an ionizing laser wavelength of $337.5 \mathrm{~nm}$. We have not scanned further due to the DCM dye laser tuning range, and actual onset could be much lower than this value. In contrast, the $\left(\mathrm{C}_{10} \mathrm{H}_{8}\right)_{3}^{+}$spectrum is characterized by a more rapidly rising curve at the onset region. This allows one to determine the onset to be $\approx 62,500 \mathrm{~cm}^{-1}$, which is still far below the corresponding onset for the monomer (i.e., $\overline{8}_{0}^{1} \rightarrow \overline{8}_{0}^{+} @ 66,050 \mathrm{~cm}^{-1}$ ) obtained under the same extraction field condition $(315 \mathrm{~V} / \mathrm{cm})$. The photoionization 


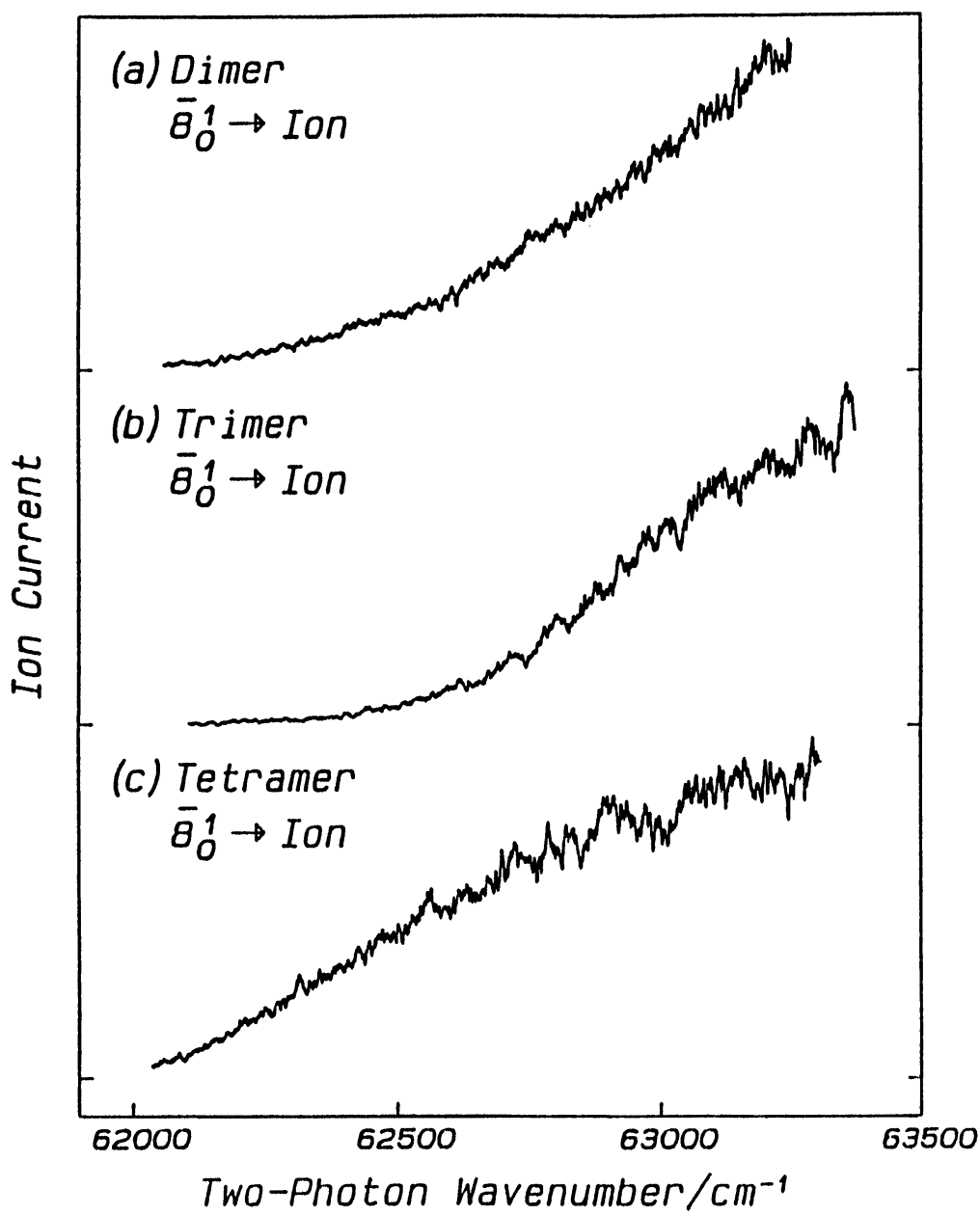

Figure 4 Comparison of two-color photoionization efficiency curves taken for (a) $\left(\mathrm{C}_{10} \mathrm{H}_{8}\right)_{2}^{+}$, (b) $\left(\mathrm{C}_{10} \mathrm{H}_{8}\right)_{3}^{+}$, and (c) $\left(\mathrm{C}_{10} \mathrm{H}_{8}\right)_{4}^{+}$. In each case, the pump laser is fixed at the $\overline{8}_{0}^{1}$ intermediate of the neutral cluster while the ionizing laser, time-delayed by $200 \mathrm{~ns}$, is scanned in the wavelength range of 336.25 $322.5 \mathrm{~nm}$. These spectra were not corrected for the intensity variation of the ionizing laser (see Figure 8 for the laser power spectrum).

curve for $\left(\mathrm{C}_{10} \mathrm{H}_{8}\right)_{2}^{+}$also shows a gradually rising onset similar to that observed for $\left(\mathrm{C}_{10} \mathrm{H}_{8}\right)_{4}^{+}$. Another important observation is that the ionization efficiency curve of $\left(\mathrm{C}_{10} \mathrm{H}_{8}\right)_{4}^{+}$obtained subsequent to excitation through the $\mathrm{S}_{1}$ origin $\left(\right.$ at $\left.31,911 \mathrm{~cm}^{-1}\right)$ is nearly identical to the corresponding curve for $\left(\mathrm{C}_{10} \mathrm{H}_{8}\right)_{3}$, both featuring a relatively clear ionization onset at $\approx 62,200 \mathrm{~cm}^{-1}$. This behavior differs markedly from the situation in the $\overline{8}_{0}^{1}$ region where the dissimilarity between the ionization curves for $\left(\mathrm{C}_{10} \mathrm{H}_{8}\right)_{3}^{+}$and $\left(\mathrm{C}_{10} \mathrm{H}_{8}\right)_{4}^{+}$is evident. 


\section{B. Ionization of trimer through $7_{0}^{1}$ intermediate}

In the $S_{1}$ spectrum of the naphthalene monomer, the $7_{0}^{1}$ band $\left(\right.$ at $\left.32,931 \mathrm{~cm}^{-1}\right)$ is the first vibronic transition accessible by one-color $(1+1)$ ionization. The most remarkable result for the corresponding excitation of $\left(\mathrm{C}_{10} \mathrm{H}_{8}\right)_{3}$ is that the two-color method gives rise to highly efficient ionization with respect to the one-color method. Figure 5 compares the ionization excitation spectra encompassing this band, recorded by the one- and two-color methods, plotted as a shift with respect to its $S_{1}$ origin at $31,977 \mathrm{~cm}^{-1}$. The ionization laser, delayed by $200 \mathrm{~ns}$, was fixed at $320 \mathrm{~nm}$ to record the two-color spectrum. As discussed earlier, ${ }^{5}$ the $7{ }_{0}^{1}$ transition consists of two peaks (at 32,843 and $32,848 \mathrm{~cm}^{-1}$ ) that can be assigned either to an exciton splitting or to an inequivalent site splitting. The two-color spectrum clearly resolves the splitting $\left(\approx 4.9 \mathrm{~cm}^{-1}\right)$ having a peak intensity ratio of $\approx 1: 2$.

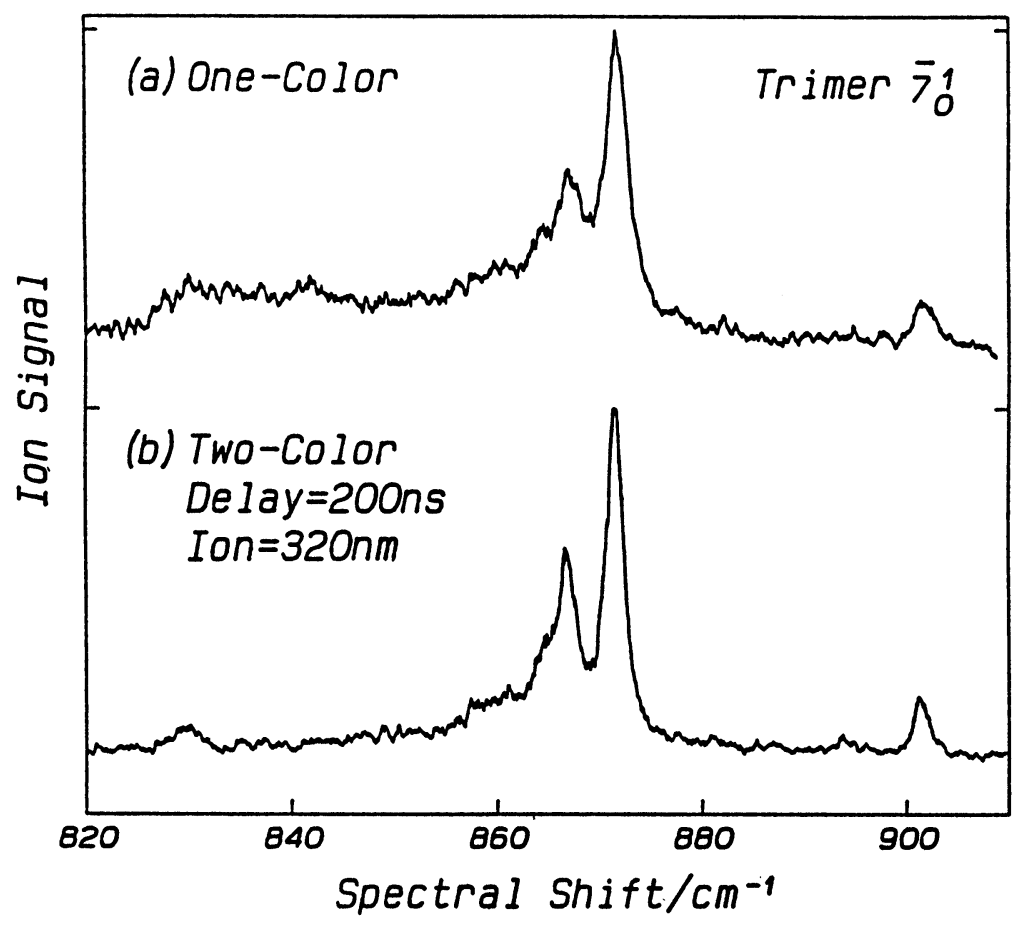

Figure 5 Comparison of R2PI spectra of the naphthalene trimer in the $\overline{7}_{0}^{1}$ region, recorded by (a) the one-color method and (b) the two-color method. The two-color spectrum is taken when the ionising laser (fixed at $320 \mathrm{~nm}$ ) fires with a 200-ns time delay. The spectral shift is with respect to the $S_{1}$ origin of the trimer at $31,977 \mathrm{~cm}^{-1}$.

As reported previously, ${ }^{6}$ excimer formation occurs very slowly subsequent to excitation of the trimer into the $7_{0}^{1}$ intermediate, thus producing a clear build up of the excimer fluorescence. The formation time has been estimated to be $32 \mathrm{~ns}$. We present here direct evidence that the enhancement of the two-color ionization observed for 
this excitation is associated with the excimer formation. Figure 6 displays the twocolor TOF spectra obtained by varying the pump-probe delay from 3 to $225 \mathrm{~ns}$. The $\left(\mathrm{C}_{10} \mathrm{H}_{8}\right)_{3}^{+}$signal resulting from the $7_{0}^{1}$ intermediate excitation shows a clear buildup at short delays and reaches a maximum at a delay time of approximately $75 \mathrm{~ns}$. The signal then decays with a slow lifetime which coincides well with the decay lifetime of the excimer fluorescence $(340 \mathrm{~ns}){ }^{6}$ The temporal behaviors of the $\left(\mathrm{C}_{10} \mathrm{H}_{8}\right)_{3}^{+}$ion signal and the excimer fluorescence signal are compared in Figure 7. The interpretation of the data is straightforward. The two-color signal is associated with ionization from the trimer cluster undergoing a structural rearrangement into an excimer geometry. The evolution of the $\left(\mathrm{C}_{10} \mathrm{H}_{8}\right)_{3}^{+}$signal indicates that the ionizing transition occurs more efficiently from the excimer than from the initially excited vdW cluster. Thus, the maximum signal appearing at a $75 \mathrm{~ns}$ delay corresponds well to that of the excimer fluorescence signal which occurs at $80 \mathrm{~ns}$. This temporal behavior is quite different from that for $\left(\mathrm{C}_{10} \mathrm{H}_{8}\right)_{3}^{+}$obtained through the $\overline{8}_{0}^{1}$ intermediate excitation (at $\left.32,374 \mathrm{~cm}^{-1}\right)$, where no such evolution is observed. The $\left(\mathrm{C}_{10} \mathrm{H}_{8}\right)_{3}^{+}$ion signal decays with a single exponential lifetime of $\approx 300 \mathrm{~ns}$, which is consistent with the absence of excimer formation at this level. The trimer gives rise to vdW-type fluorescence having a $290-$ ns lifetime. ${ }^{6}$

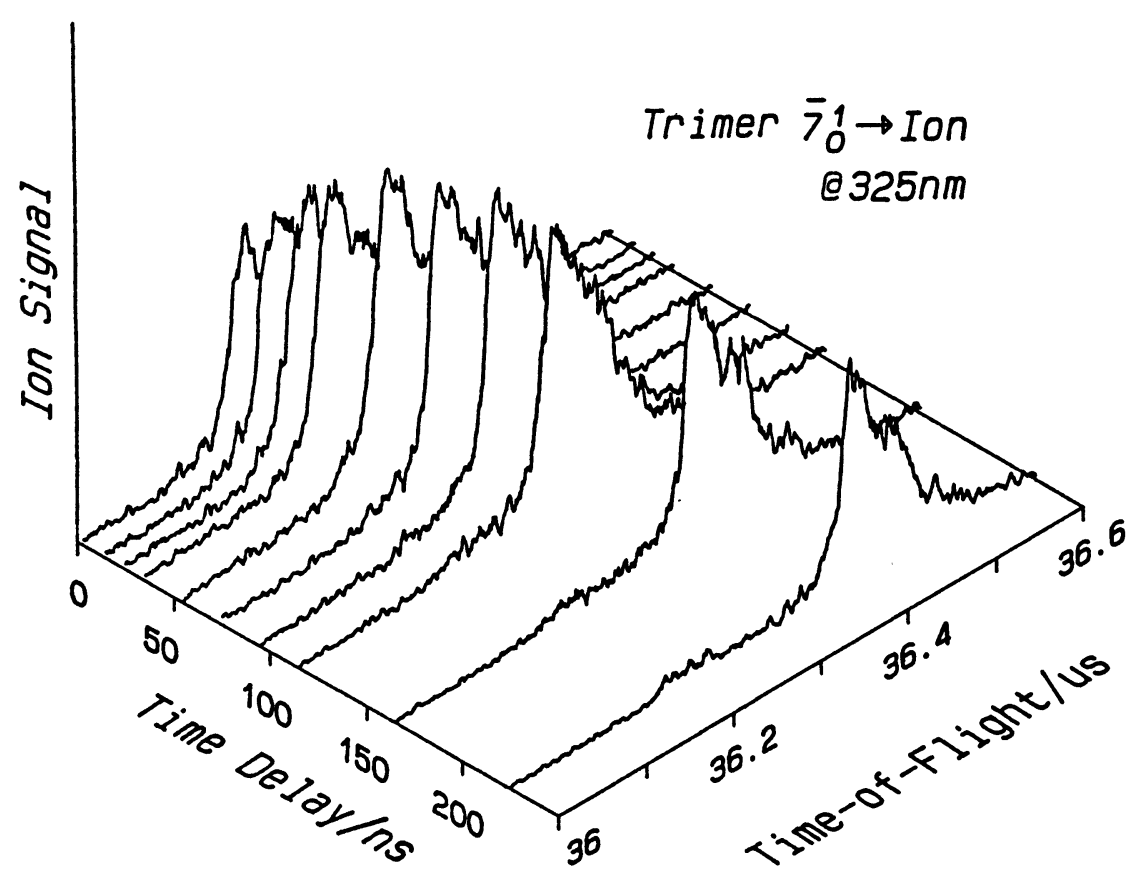

Figure 6 Two-color TOF mass spectra of the naphthalene trimer ion $\left(\mathrm{C}_{10} \mathrm{H}_{8}\right)_{3}^{+}$obtained as a function of the time delay between the pump and ionizing laser pulses. The trimer cluster is excited at the $\overline{7}_{0}^{1}$ transition $\left(32,848 \mathrm{~cm}^{-1}\right)$ shown in Figure 5, and then ionized at $325 \mathrm{~nm}$. 


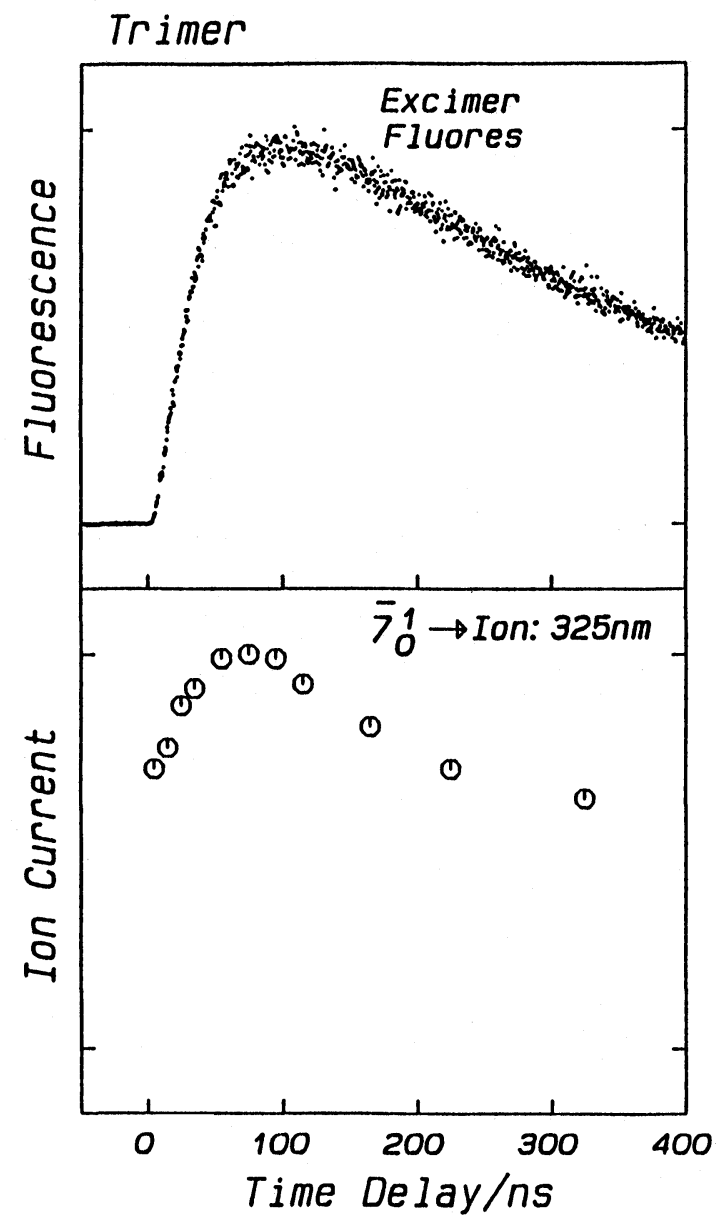

Figure 7 Comparison of temporal behaviors for the two-color signal of $\left(\mathrm{C}_{10} \mathrm{H}_{8}\right)_{3}^{+}($lower panel) and for the excimer fluorescence (upper panel). The two-color signal is plotted as a function of the time delay between the pump laser pulse, tuned to the $\overline{7}_{0}^{1}$ transition of the trimer, and the ionizing laser pulse at $325 \mathrm{~nm}$. The excimer fluorescence resulting from the same pump laser excitation is detected at $>400 \mathrm{~nm}$.

The time-resolved photoionization spectra of $\left(\mathrm{C}_{10} \mathrm{H}_{8}\right)_{3}^{+}$recorded with pump-probe delays of 3 and $115 \mathrm{~ns}$, are compared in Figure 8. It is important to note that ionization efficiency evolves in the entire spectral region as the delay is increased from 3 to $115 \mathrm{~ns}$. Comparing to the early time spectrum, which exhibits a relatively clear onset at $62,800 \mathrm{~cm}^{-1}$, the spectrum obtained at $115 \mathrm{~ns}$ enhances a long tail extending to a lower energy. Note also that the $115 \mathrm{~ns}$ spectrum resembles the photoionization curve for $\left(\mathrm{C}_{10} \mathrm{H}_{8}\right)_{4}^{+}$obtained at the $\overline{8}_{0}^{1}$ intermediate excitation (Figure 4(c)). These findings are consistent with the spectral evolution observed in the TOF spectra (Figure 6), confirming that the excimer formation is responsible for the enhancement of the two-color signal over the one-color signal. 


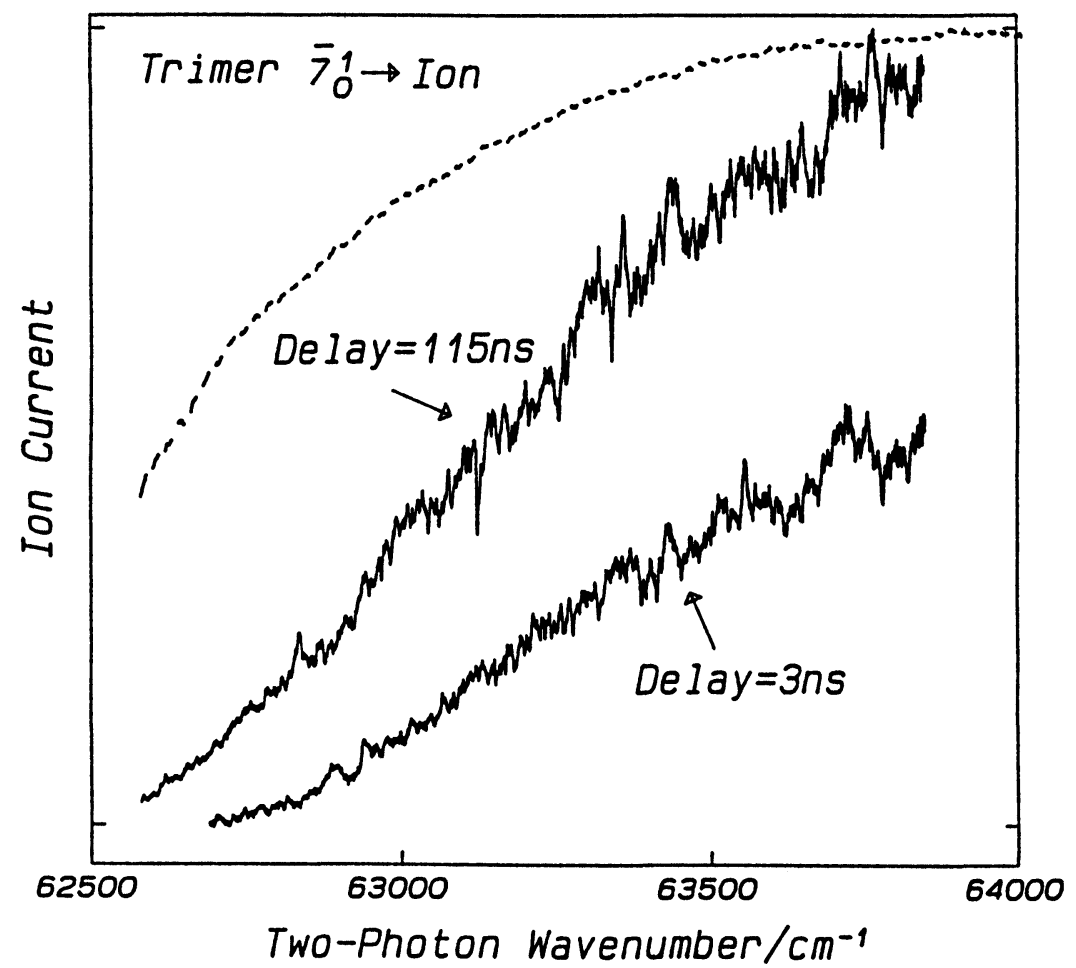

Figure 8 Comparison of two-color photoionization efficiency spectra of $\left(\mathrm{C}_{10} \mathrm{H}_{8}\right)_{3}^{+}$, recorded at pumpprobe delays of $3 \mathrm{~ns}$ and $115 \mathrm{~ns}$. In each case, the pump laser is fixed at the $7_{0}^{1}$ transition of the trimer $\left(32,848 \mathrm{~cm}^{-1}\right)$ while the ionizing laser is scanned in the wavelength range of $336.25-322.5 \mathrm{~nm}$ (which corresponds to the total energy range of $\left.62,600-63,850 \mathrm{~cm}^{-1}\right)$. The dotted line represents the intensity variation of the ionizing laser.

\section{DISCUSSION}

The emphasis of this paper follows the two major directions; first, the mass spectroscopic detection and identification of the excimers produced subsequent to excitation of $\left(\mathrm{C}_{10} \mathrm{H}_{8}\right)_{\mathrm{n}}$, and second, the enhancement of cluster ionization by the excimer formation.

\section{A. Photoionization detection of the naphthalene excimers}

The results of the present photoionization studies indicate unequivocally the absence of monomer evaporation upon excimer formation. The evidence is that the temporal behavior of the two-color signal $\left(\mathrm{C}_{10} \mathrm{H}_{8}\right)_{4}^{+}$, obtained through excitation of the corresponding neutral cluster, coincides well with that of the excimer fluorescence. Furthermore, the $\left(\mathrm{C}_{10} \mathrm{H}_{8}\right)_{3}^{+}$signal resulting from the $7_{0}^{1}$ intermediate excitation is characterized by a buildup which matches the rise time of the excimer fluorescence. 
These observations indicate that the excimer formation process is associated with a structural rearrangement of the initially excited cluster, and loss of ground-state molecules is not occurring.

Then, the question arises whether the electronic excitation of the excimer is localized on a specific pair of naphthalene molecules, i.e., $\left(\mathrm{C}_{10} \mathrm{H}_{8)_{2 \times(\mathrm{C}}{ }_{10}} \mathrm{H}_{8)_{n}}-2\right.$, or is shared among all the cluster constituents, i.e., $\left(\mathrm{C}_{10} \mathrm{H}_{8}\right)_{\mathrm{n}}{ }^{*}$. However, there are some indications from previous experiments and calculations on these excimers and other systems that the latter possibility is unlikely. In particular, we have recently demonstrated that the naphthalene excimer produced in a parent cluster can be further excited at wavelengths between 500 and $900 \mathrm{~nm},{ }^{9}$ which results in rapid dissociation. The resulting photofragments are naphthalene monomers, at least one of which being electronically excited and thus emitting the UV fluorescence of naphthalene. If all the constituent molecules involved in the excimer were sharing the electronic excitation, most of the photofragments would be expected to be in the electronically ground state. In addition, the two different excimers, one resulting from $\left(\mathrm{C}_{10} \mathrm{H}_{8}\right)_{3}$ and the other from $\left(\mathrm{C}_{10} \mathrm{H}_{8}\right)_{4}$, show the same photodissociation behavior in the visible and near IR wavelength regions; the maximum photodissociation yield occurs at approximately $700 \mathrm{~nm}$, irrespective of the excimer identity. From these considerations, we conclude that the excimer involves an excitation-localized dimer core having an interplanar separation much less than those with other ground-state molecules, i.e., $\left.\left(\mathrm{C}_{10} \mathrm{H}_{8}\right)_{2} *\left(\mathrm{C}_{10} \mathrm{H}_{8}\right)_{n}-2\right\}$. This leads us to adopt a model for the excimer photodissociation that the excimer core $\left(\mathrm{C}_{10} \mathrm{H}_{8}\right)_{2}$ * absorbs strongly at $700 \mathrm{~nm}$, re-sulting in the formation of photofragments: $\left(\mathrm{C}_{10} \mathrm{H}_{8}\right)_{2} *\left(\mathrm{C}_{10} \mathrm{H}_{8}\right)_{\mathrm{n}-2} \rightarrow\left(\mathrm{C}_{10} \mathrm{H}_{8}\right) *+(\mathrm{n}-1)\left(\mathrm{C}_{10} \mathrm{H}_{8}\right)$.

Given that the excimer formation proceeds in the absence of monomer evaporation, it is necessary to consider the relationship between excimer formation and vibrational relaxation. The dynamical problem involved appears to be analogous to those discussed for explaining the excimer formation in molecular crystals. ${ }^{14-17}$ The excimer produced in aromatic crystals (e.g. pyrene and perylene) is also described as a pair of molecules sharing electronic excitation. Its equilibrium geometry must involve large structural displacement from the crystal configuration. The excimer pair has been treated as a giant molecule having internal coordinates which change substantially upon excimer formation. ${ }^{16}$ The vibrational frequencies of these internal coordinates may be high enough to be treated as intramolecular vibrations. Thus, the initial excitation can be trapped by the excimer, and randomized quickly throughout the vibrational degrees of freedom of the excimer coordinates. The possibility of vibrational relaxation in the excimer potential well has been attributed either to an anharmonicity of the potential or to a coupling with lattice vibrations. ${ }^{15,17}$ Obviously, a more rigorous treatment for the structural rearrangement is necessary for a detailed understanding of excimer formation. The information presented herein should facilitate this kind of consideration. 


\section{B. Ionization mechanism of aromatic clusters}

The photoionization results of the naphthalene clusters undergoing excimer formation provide more important information on the ionization mechanism of small aromatic clusters. There are two plausible mechanisms for explaining some characteristics of multiphoton ionization observed in these clusters.

\section{Cooperative ionization mechanism}

Several aromatic crystals exhibit strong evidence of autoionization from higher excited state generated by two-photon absorption, suggesting the occurrence of sequential two-step ionization involving diffusion and annihilation of Frenkel excitons. ${ }^{18,19}$ Schriver et al. ${ }^{10}$ first proposed an analogous mechanism to account for the highly efficient ionization observed for large benzene clusters $(n>20)$. Subsequently, Wessel ${ }^{11}$ reported that the two-photon ionization enhancement for the naphthalene trimer differs markedly from typical ionization behaviors observed for other clusters. The two-color ionization signal was detected whenever the ionizing laser beam was tuned to the vicinity of the $S_{1}$ vibronic level $\left(\overline{8}_{0}^{1}\right)$ excited by the pump laser. This behavior was taken as direct evidence for a biexcitonic autoionization process. Apparently, this mechanism cannot be reconciled with our observation that the trimer excited at the same level ionizes via conventional $\left(1+1^{\prime}\right)$ ionization.

Extensive analyses of the $S_{1}$ spectra of the naphthalene clusters were reported by Wessel and Syage. ${ }^{4}$ On the basis of resonance interaction calculations, they predicted the most plausible magnitude of exciton interactions to be $10<|\beta|<$ $20 \mathrm{~cm}^{-1}$ for the trimer $\overline{8}_{0}^{1}$ transition. However, there are indications from our results that such excitonic interactions are not a significant effect in the initially excited state of this cluster. For example, the $\overline{8}_{0}^{1}$ band of $\left(\mathrm{C}_{10} \mathrm{H}_{8}\right)_{3}$ reveals a doublet structure having an $8 \mathrm{~cm}^{-1}$ splitting (shown in Figure 3(b)), while no corresponding splitting is observed for the isotopically mixed trimer $\left(\mathrm{C}_{10} \mathrm{H}_{8}\right)_{1} *\left(\mathrm{C}_{10} \mathrm{D}_{8}\right)_{2}$ in which the excitation is carried out into the $\left(\mathrm{C}_{10} \mathrm{H}_{8}\right)_{1}$ moiety. ${ }^{20}$ One possible explanation for the splitting observed for the pure trimer is due an exciton interaction between a pair of the constituents, thus explaining the absence of such a splitting for the mixed trimer. On the basis of this assumption, we obtain an upper limit for the pairwise interaction to be $|\beta| \leq 4 \mathrm{~cm}^{-1}$, neglecting inequivalent site effects. It is also likely that inequivalent site effects contribute to the observed splitting. Analogous isotope dependence has been observed for the $\overline{8}_{0}^{1}$ multiplet of $\left(\mathrm{C}_{10} \mathrm{H}_{8}\right)_{4}{ }^{20}$ While these considerations are strongly against the exciton annihilation mechanism, it may well be that both mechanisms contribute to the trimer ionization through the $\overline{8}_{0}^{1}$ intermediate and the biexcitonic autoionization process becomes important at higher fluences of the pump laser. 


\section{Photoionization via excimer formation}

The possibility of a structural relaxation effect on the photoionization of the benzene dimer was discussed before, first by Hopkins et al. ${ }^{7}$ and subsequently by Grover et al. ${ }^{21}$ The present results unequivocally indicate that excimer formation involving a large structural relaxation is responsible for the enhancement of ionization efficiency for the naphthalene clusters. This mechanism explains the observation that the ionization efficiency curve for $\left(\mathrm{C}_{10} \mathrm{H}_{8}\right)_{4}^{+}$obtained through the $\overline{8}_{0}^{1}$ intermediate extends to a much lower energy than that for $\left(\mathrm{C}_{10} \mathrm{H}_{8}\right)_{3}^{+}$. The difference reflects that the $\left(\mathrm{C}_{10} \mathrm{H}_{8}\right)_{4}$ cluster excited at $\overline{8}_{0}^{1}$ rearranges rapidly into an excimer geometry while no significant structural relaxation occurs for $\left(\mathrm{C}_{10} \mathrm{H}_{8}\right)_{3}$ at the corresponding excitation. On the basis of the fluorescence measurements, we can estimate an upper limit for the excimer formation time in $\left(\mathrm{C}_{10} \mathrm{H}_{8}\right)_{4}$ to be $<1 \mathrm{~ns}$. This implies that, in the nanosecond pumpprobe scheme employed in this study, the ionizing transition always takes place from the excimer state rather than from the initially excited state. In other words, the two-color technique is probing an adiabatic ionization process from the excimer state. For the $\left(\mathrm{C}_{10} \mathrm{H}_{8}\right)_{3}$ cluster, the ionizing photon can interact with the initially excited state with a vdW geometry, thus resulting in a nearly vertical ionization.

More direct evidence which strongly favors the ionization mechanism involving excimer formation derives from the results of the time-resolved two-color ionization for $\left(\mathrm{C}_{10} \mathrm{H}_{8}\right)_{3}$ through the $7_{0}^{1}$ intermediate excitation. Excitation into this band produces anomalously slow evolution of the two-color ionization signal, which corresponds well to the rise time of the excimer fluorescence. This behavior is expected based on the observation that the initially excited vdW cluster rearranges to the excimer with a 32 ns lifetime. Thus, the two-color signals, obtained at pump-probe delays shorter than the excimer formation time, can be assigned to ionization from the vdW state while those occurring at longer delays are associated with the excimer state. Such temporal evolution is more pronounced at ionization wavelengths longer than $330 \mathrm{~nm}$ (which corresponds to a total energy of $62,800 \mathrm{~cm}^{-1}$ ), where virtually no ionization signal is observed for the early time $(3 \mathrm{~ns})$ spectrum shown in Figure 8.

All of these results indicate that the occurrence of excimer formation can significantly lower the ionization thresholds of these species, thus leading to the enhancement of their ionization efficiencies. This behavior is most likely associated with larger Franck-Condon factors for the ionizing transition from the excimer state with respect to those from the vdW excited state. As discussed above, the excimer produced in these clusters are likely to have a dimer core to which electronic excitation is confined, i.e., $\left(\mathrm{C}_{10} \mathrm{H}_{8}\right)_{2} *\left(\mathrm{C}_{10} \mathrm{H}_{8}\right)_{\mathrm{n}-2}$. Similarly, the stability of the naphthalene cluster ions can be explained by a charge-localized structure involving a strongly bound dimer ion core, surrounded by neutral molecules which are less strongly bound. Based on the assumption of the structural similarity between the excimer and cluster ion, the Franck-Condon factors associated with the ionizing transition are expected to be higher for the excimer than for the vdW cluster.

One apparent problem is that the long tailing of the ion current observed for the excimer ionization is not what would be expected for the case of such close structural 
similarity. The clue to understanding the gradual tailing, a characteristic signature of Franck-Condon difficulties, lies in the difference between the absorption spectra of the excimer and dimer ion. The transition between the bound excimer state and the repulsive excimer state (i.e., $\psi^{*} \psi \pm \psi \psi^{*}$, where $\psi$ and $\psi^{*}$ are the ground- and second excited-state wavefunctions of the neutral molecule, respectively), obtained by the photodissociation technique, exhibits a maximum at $\approx 1.8 \mathrm{eV} .{ }^{9}$ The corresponding transition for the dimer ion (i.e., $\psi^{+} \psi \pm \psi \psi^{+}$, where $\psi^{+}$is the ground-state wavefunction of the ion) is observed at $\approx 1.2 \mathrm{eV}$ in solutions. ${ }^{22}$ This difference presumably reflects that the excimer core $\left(\mathrm{C}_{10} \mathrm{H}_{8}\right)_{2}{ }^{*}$ is more strongly bound with respect to the dimer ion $\left(\mathrm{C}_{10} \mathrm{H}_{8}\right)_{2}^{+}$. The interplanar separation of the naphthalene excimer has been estimated to be $3.2 \AA,{ }^{23}$ while that of $\left(\mathrm{C}_{10} \mathrm{H}_{8}\right)_{2}^{+}$is expected to be larger than this value. A schematic diagram pertinent to the excimer formation and ionization of the naphthalene clusters is shown in Figure 9.

The proposed mechanism for the ionization enhancement is in agreement with the earliest interpretation of the two-color ionization results on the benzene dimer by Hopkins et al. ${ }^{7}$ The remarkable enhancement of the ionization over the one-color signal was explained by invoking excimer formation occurring within several picoseconds. They described that the dimer, most probably having a T-shaped structure, rearranges to a parallel stacked excimer structure prior to ionization. Nevertheless, no excimer fluorescence was detected subsequent to excitation of the dimer into the $S_{1}$ electronic origin. ${ }^{8}$ Based on this result, they suggested that the benzene excimer has a sandwiched geometry with $\mathrm{D}_{6 \mathrm{~h}}$ symmetry and thus the transition to the ground state is forbidden. A similar ionization behavior was obtained for the benzene dimer and trimer when excited into the $S_{2}$ state. ${ }^{24}$ The existence of a long-lived (120-140 ns) species, which cannot be observed for the corresponding monomer excitation, was attributed to excimer formation.

In the case of naphthalene trimer and tetramer discussed above, the unique relationship between the fluorescence and ionization behaviors provides direct evidence for a large geometrical change that can be associated with the excimer formation. Analogous arguments will apply to the naphthalene dimer. Its ionization efficiency curve (shown in Figure 4(a)) is characterized by a gradual onset, indicating that the ionization occurs from the excimer state, consistent with the fluorescence measurement. In contrast to the benzene dimer, the $S_{1}$ spectrum of the naphthalene dimer is dominated by broad structureless bands. This suggests that the $S_{1}$ dimer has a stable geometry which differs significantly from the ground-state geometry. One possible geometry for the $S_{1}$ dimer is a parallel displaced structure which can rearrange into an overlapped excimer geometry over a low energy barrier. This structure suggests the existence of an extensive interstate coupling of the vdW excited state with the excimer state, thus explaining the highly efficient excimer formation for the dimer. However, the broad excitation spectra cannot be explained as arising from direct excitation into the excimer state, since the vibrational frequencies derived from the band center positions are essentially identical to those of the monomer. Thus, the broadening may be associated with unresolved progressions in low-frequency intermolecular vibrations, with additional contributions due to a rapid excimer formation rate. 


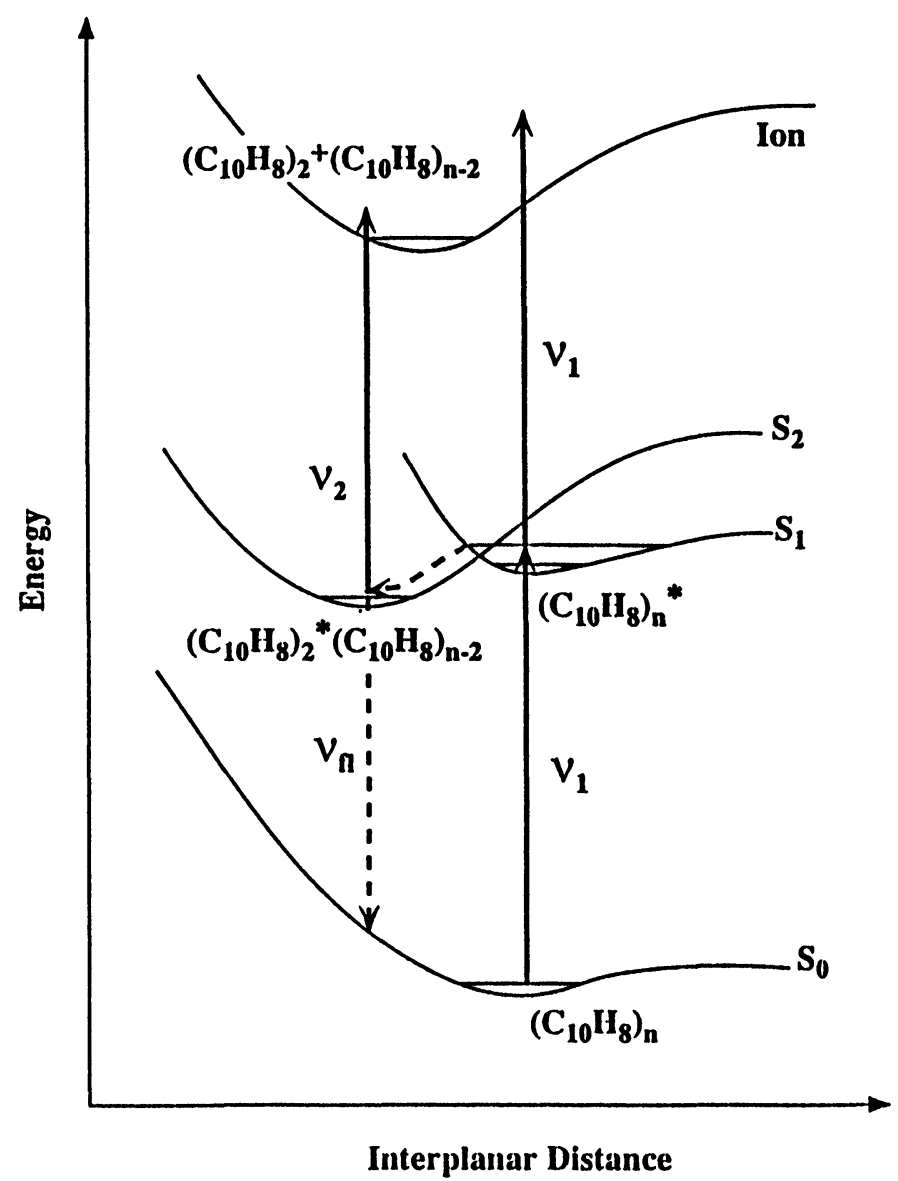

Figure 9 Schematic diagram illustrating the two-photon ionization processes in naphthalene clusters $\left(\mathrm{C}_{10} \mathrm{H}_{8}\right)_{\mathrm{n}}$. One-color $\left(2 \nu_{1}\right)$ ionization occurs via $\mathrm{S}_{1}$ vibronic levels of the parent clusters $\left(\mathrm{C}_{10} \mathrm{H}_{8}\right)_{\mathrm{n}}{ }^{*}$ while two-color $\left(v_{1}+v_{2}\right)$ ionization arises from the excimer state $\left(\mathrm{C}_{10} \mathrm{H}_{8}\right)_{2} *\left(\mathrm{C}_{10} \mathrm{H}_{8}\right)_{\mathrm{n}-2}$. The resulting ion is composed of a dimer ion core and neutral molecules, $\left(\mathrm{C}_{10} \mathrm{H}_{8}\right)_{2}^{+}\left(\mathrm{C}_{10} \mathrm{H}_{8}\right)_{\mathrm{n}-2}$.

In conclusion, we have provided direct spectroscopic evidence for structural relaxation occurring in the naphthalene clusters when excited into the $S_{1}$ vibronic levels. Based on the two-color photoionization results, the process has been identified as a structural rearrangement of the initially excited cluster having a vdW geometry into an excimer geometry, thus involving no monomer evaporation. The resulting excimer is assigned to an excited-state cluster having a strongly bound dimer core surrounded by weakly bound ground-state naphthalenes. This study also demonstrates that the excimer ionizes with a higher efficiency with respect to the initially prepared vdW cluster. This behavior is explained in terms of the structural similarity between the excimer and cluster ion. We suggest that the occurrence of excimer formation also explains the observation of photoionization enhancement in other aromatic cluster. 
Currently, we are trying to search for the possibility of excimer formation in the benzene dimer.

\section{Acknowledgments}

This paper is dedicated with gratitude to Professor Mitsuo Ito, whose quantum chemistry lectures at Tohoku University kindled the author's interest in the area of molecular spectroscopy. The author also thanks Professor E. C. Lim for valuable discussions.

\section{References}

1. For example, see Microclusters, edited by S. Sugano, Y. Nishina, and S. Ohnishi (Springer, New York, 1987); Atomic and Molecular Clusters, edited by E. R. Bernstein (Elsevier, Amsterdam, 1990).

2. D. M. Hanson. J. Chem. Phys., 52, 3409 (1970).

3. J. E. Wessel and J. A. Syage. J. Chem. Phys., 89, 5962 (1988).

4. J. E. Wessel and J. A. Syage. J. Phys. Chem., 94, 737 (1990).

5. H. Saigusa, S. Sun and E. C. Lim. J. Phys. Chem., 96, 2083 (1992).

6. H. Saigusa, S. Sun and E. C. Lim. J. Chem. Phys., 97, 9072 (1992).

7. J. B. Hopkins, D. E. Powers, and R. E. Smalley. J. Phys. Chem., 85, 3739 (1981).

8. P. R. R. Langridge-Smith, D. V. Brumbaugh, C. A. Haynam and D. H. Levy. J. Phys. Chem., 85, 3742 (1981).

9. H. Saigusa S. Sun and E. C. Lim. J. Phys. Chem., 96, 10099 (1992).

10. K. E. Schriver, M. Y. Hahn and R. L. Whetten. Phys. Rev. Lett., 59, 1906 (1987).

11. J. E. Wessel. Phys. Rev. Lett., 64, 2046 (1990).

12. S. M. Beck, M. G. Liverman, D. L. Monts and R. E. Smalley. J. Chem. Phys., 70, 232 (1979).

13. M. Stockburger, H. Gatterman, and W. Klusmann. J. Chem. Phys., 63, 4519 (1975).

14. V. Yakhot, M. D. Cohen and Z. Ludmer, Z. Adv. Photochem., 11, 489 (1979).

15. M. D. Cohen and V. Yakhot. Chem. Phys., 5, 27 (1974).

16. D. P. Craig, L. Dissado and S. H. Walmsley. Chem. Phys., 46, 87 (1980).

17. L. A. Dissado and S. H. Walmsley. Chem. Phys. Lett., 87, 74 (1982); Chem Phys., 86, 375 (1984).

18. C. L. Braun. Phys. Rev. Lett., 21, 215 (1968).

19. S. Arnold, M. Pope and T. K. T. Hsieh. Phys. Status Solidi, B 94, 263 (1979).

20. H. Saigusa et al., (in preparation).

21. J. R. Grover, E. A. Walters and H. Baumgärtel. J. Phys. Chem., 93, 7534 (1989).

22. B. Badger, B. Brocklehurst and R. D. Russell. Chem. Phys. Lett., 1, 122 (1967).

23. T. Azumi, A. T. Armstrong and S. P. McGlynn. J. Chem. Phys., 41, 3839 (1964).

24. H. Shinohara and N. Nishi. J. Chem. Phys., 91, 6743 (1989). 